\title{
Characterizing the Nearby Solar-Type Stars
}

\author{
D. R. Soderblom and J. R. King \\ Space Telescope Science Institute \\ 3700 San Martin Drive \\ Baltimore MD 21218
}

\begin{abstract}
We present a brief discussion of solar-type stars, what is known about them, why they are ideal targets for precise radial-velocity observations, and some open questions.
\end{abstract}

\section{Context}

This symposium on precise radial velocities has emphasized solar-type stars (STSs) because they are so well-suited to that type of observation:

- STSs have lots of intrinsically narrow absorption lines.

- Old STSs are among the quietest of stars photometrically.

- STSs offer a nice balance between luminosity and frequency of occurrence (IMF) so that there are reasonable numbers of bright ones.

- Earlier type stars (about F5V and earlier) have few lines and those lines tend to be broad.

- Main sequence stars later than $\mathrm{G}$ are faint, making radial velocity precision costly.

- Evolved stars appear to exhibit radial velocity variability that is intrinsic to the star. This is interesting, but it hampers detecting low-mass companions.

These properties account for the fact that the sub-stellar bodies found so far have been around old STSs. This is not because such bodies do not exist elsewhere, but instead because we can at present only detect them in the environs of STSs. Ultimately we will want to know about such objects in all environments, but the difficulty of detection means we will settle for whatever we can get. This difficulty also means that for the present it is all right to apply whatever knowledge of the stars we have that might improve the chances of detection.

This ability to detect low-level RV variability in STSs makes such stars ideal for addressing several astrophysical problems: 1) finding planets and other low-mass bodies; 2) studying multiplicity in general as a star formation issue; and 3) stellar kinematics as a tool for understanding Galactic processes.

What does "solar-type" mean? The phenomena that we see on the Sun that make it so interesting are all related to the existence of the Sun's convective 
envelope. Convection and rotation together (plus differential rotation) lead to the magnetic dynamo. The visible manifestations of the magnetic field include spots, faculae, flares, and so on. The magnetic field can hold on to the ionized solar wind beyond the solar surface, and that leads to angular momentum loss. Thus STSs spin down as they get older, and their activity decreases.

But why care about lists of stars and databases? One good example is provided by 51 Pegasi, now so well known as the first instance of an extra-solar planet. One reason Mayor \& Duquennoy were first to find the companion to 51 Peg is that Marcy \& Butler took their sample from the Bright Star Catalogue, where $51 \mathrm{Peg}$ is classed as a G2.5IVa star. $51 \mathrm{Peg}$ is actually a main sequence star, and we can do better.

\section{Observations of Solar-Type Stars}

How well can we determine the fundamental properties of an STS? By "fundamental properties" we mean those qualities that lead to physical understanding of an object. It may be possible to determine a star's color, say, to $0.1 \%$, but the inferred temperature is not ordinarily better than to about $100 \mathrm{~K}$ due to significant systematic effects.

For instance, a star's mass is probably its single most important parameter, but measuring masses as well as $1 \%$ is rare, especially for well-separated systems where we can be confident that no physical interactions between the companions are taking place. The general nature of the HR diagram tells us that STSs are common in the solar neighborhood, but we do not know true masses of STSs except in a very few instances.

A star's composition is also a critical parameter, and here the situation is substantially better. With STSs we have the great advantage of being able to work differentially relative to the Sun itself, and we know solar parameters very well indeed. We usually parameterize composition as $[\mathrm{Fe} / \mathrm{H}]$ because ironpeak elements are fairly easy to analyze, but the story of stellar abundances is much more complex than that. Even so, there are systematic effects that limit our ability to measure $[\mathrm{Fe} / \mathrm{H}]$ to better than about 0.1 dex, even for stars that are near-solar twins. Perhaps most limiting in these determinations are temperatures, but Gray (1994a, 1994b) has now shown how to detect temperature differences of as little as $6 \mathrm{~K}$ between stars. His method depends on line ratios and is purely spectroscopic, so a bona fide comparison to the Sun is possible. However, good spectroscopic resolution and very high signal-to-noise ratios are needed, and the method has not been applied except in a few cases.

Age is also important to how a star appears to us. Our Galactic disk is $\sim 10 \mathrm{Gyr}$ old, so the Sun, at $4.5 \mathrm{Gyr}$, is near the average age. The median age of stars in the solar neighborhood is more like 3 Gyr because of "disk heating," so the Sun is a little on the old side. We know the Sun's age on fundamental grounds, but the ages of other stars must be inferred. One indicator of age for STSs is activity, usually measured using the reversals in the centers of the Ca II $\mathrm{H}$ and $\mathrm{K}$ lines. The youngest stars of the solar neighborhood have activity levels about 10 times that of the Sun, but most stars are old and have correspondingly low activity (Vaughan \& Preston 1980). 
Another important, if subtler, property of a star is its rotation. As we described, rotation and age are intimately related for STSs, but there is not a strict one-to-one relationship between the two. If nothing else, we are confident that stars are born with a range of angular momenta. The rate at which angular momentum is lost is greater for stars that rotate faster, so there is convergence in rotation rates with time. There is some evidence that the radiative cores of stars decouple from the convective envelopes when stars are young, with the cores spinning faster, but there is no concrete evidence of this yet.

\section{Defining Samples of STSs}

In pre-Hipparcos times, the best sample of STSs was that of Olsen $(1988,1993)$. He started with stars classed in the Henry Draper Catalogue as G0 or G5; K0 was not included because the contamination by giants would be severe. He obtained Strömgren photometry to derive temperatures and gravities. Michigan two-dimensional spectral types are now available for the southern stars. Latham and Mayor, in ongoing programs, are measuring radial velocities for the Olsen stars, with 3 to 4 measures per star being typical.

The results from Hipparcos make it easier to define samples of nearby stars, although there are problems there too. Hipparcos has measured very precise astrometry for most stars brighter than magnitude 9 in $V$. The Sun would appear to be about 9th magnitude at a distance of $60 \mathrm{pc}$, so Hipparcos can provide a volume-limited sample for about $\mathrm{G} 2 \mathrm{~V}$ and earlier. But later types get faint fast, meaning more and more incompleteness for mid- to late-G dwarfs. Also, the proper motions determined by Hipparcos can be skewed by the relatively short time baseline of the mission, and in general proper motions from the PPM are better for calculating stellar kinematics.

For our purposes, we take "solar-type" to mean stars with $(B-V)$ values from 0.50 to 1.00 (about $F 8 \mathrm{~V}$ to $\mathrm{K} 2 \mathrm{~V}$ ) that are on the main sequence (in practice, within one magnitude of a theoretical zero-age main sequence). Using this definition, there are 5458 STSs in the Hipparcos catalogue that are within 60 pc. Of these, 5047 have $\sigma_{\pi} / \pi<0.1$, meaning the luminosities are reasonably well known.

The Tycho portion of the Hipparcos mission measured magnitudes and colors for the Hipparcos stars (and more), which forms an invaluable database of very high systematic quality.

\section{Deriving Quantities}

As we mentioned, the conventional wisdom is that temperatures of stars can be determined to within about $100 \mathrm{~K}$ absolute. Relative temperatures can be determined to better precision, in general. Also, some techniques offer better possibilities. For example, Cayrel et al. (1991a, 1991b) use the wings of $\mathrm{H} \alpha$ and $\mathrm{H} \beta$ to derive temperatures to 10 to $20 \mathrm{~K}$. More recently, they applied synthetic spectra to low-resolution spectra $(R=1000, \mathrm{~S} / \mathrm{N}=50)$ to get temperatures to $75 \mathrm{~K}$, gravities to $0.2 \mathrm{dex}$, and $[\mathrm{Fe} / \mathrm{H}]$ to $0.15 \mathrm{dex}$. This is especially promising for large-scale studies. 
For the nearest stars, the accuracy of Hipparcos results has eliminated parallaxes as the source of error, but now bolometric corrections are the limiting factor, to about 0.1 magnitude. One reason this matters is the determination of the zero-age main sequence (ZAMS) for the Pleiades.

\section{Do We Understand the Basic Physics of Stellar Evolution?}

Hipparcos measured the parallaxes of members of a number of nearby open clusters, including the Hyades, Pleiades, Praesepe and $\alpha$ Persei. Most Hipparcos determinations of cluster distances agree reasonably well with "traditional" values, which are measured by comparing the cluster's main sequence to an empirical one derived from nearby stars with large parallaxes.

However, the Hipparcos measurement of the distance modulus for the Pleiades was 5.3 magnitudes, significantly different from the traditional 5.6. The Pleiades is about $100 \mathrm{Myr}$ old, so its STSs are squarely on the ZAMS. It appears to have the same composition as the Sun to within about $10 \%$. This 0.3 difference in magnitude is important. Taken at face value, it means that solar-composition ZAMS stars are $30 \%$ fainter than our models predict. Those models are built and calibrated to reproduce the present state of the Sun (radius, temperature, composition, and luminosity).

Only the Pleiades appears to have a discrepant distance, which is odd since other clusters ( $\alpha$ Persei, for instance) also have STs on the ZAMS and are also of solar composition. In a recent pair of papers (Pinsonneault et al. 1998; Soderblom et al. 1998) we have examined this Pleiades distance problem. We suspect there is a systematic effect in the Hipparcos measurements that accounts for the discrepancy, but more work is needed.

If the Hipparcos distance to the Pleiades is correct, it seems reasonable that there ought to be other stars around that are 0.3 magnitude fainter than we expect. An examination of nearby very young stars (Soderblom et al. 1998) show no such stars.

\section{Duplicity and Angular Momentum}

Companionship is a key property of a star, and especially so for STSs because of their convective envelopes. This is because a close companion can exert sufficient tidal force to cause synchronization of rotation with the orbit. This leads to much faster rotation that if the star were single and left to its own devices, and the enormous angular momentum reservoir in the orbit maintains the rapid rotation for a long time, even as angular momentum is lost at a relatively high rate. Examples of such systems are the RS CVn variables, which exhibit very strong activity.

How about more distant companions? They can disrupt planetary systems, of course, but probably do not affect the structure or evolution of the primary star. Mayor and Latham are surveying large numbers of STSs with high-precision radial velocities (to $100 \mathrm{~m} \mathrm{~s}^{-1}$ or so).

Angular momentum is a fundamental property of a star, and is at the heart of the rich phenomenology of STSs. Clearly rotation, and differences in rotation, 
lead to observable micro-properties such as the many forms of activity, but does rotation lead to differences in the macro-properties of a star? We know, for example, that STSs reach the ZAMS with a spread in surface rotation of at least a factor of 20 . STSs lose angular momentum while on the main sequence, and the dynamo mechanism includes feedback, leading to convergence in rotation rates with time. Also, rapid rotation and excess lithium may be correlated in Pleiades stars, although this is controversial. If rotation influences the rate of $\mathrm{Li}$ depletion, there is no logical feedback mechanism, and so spreads in Li should perpetuate themselves through time. Li is difficult to detect in old stars because so little Li is present, but a study of M67 (which is the age of the Sun) shows a spread in Li comparable to that in young clusters (Jones et al. 1999).

\section{Questions}

The following are some of the questions that current research on STSs is addressing:

1. Are stellar classifications based on the appearance of the spectrum (i.e., spectral types) still a quantitative tool in a time when we have good parallaxes (luminosities) and can obtain excellent high-resolution spectra easily?

Our own feeling is that the answer is "no." One unfortunate reason is that determining spectral types reliably is a skill that few are mastering, so that when the current generation is no longer doing the job spectral types will die out. Some evidence for this is seen in the pseudo-spectral types one sees published that have nothing to do with standard determinations.

2. Is there a spread in metallicity within clusters? Could such a thing occur as a result of late accretion of chondritic material, for example?

3. Do STSs have uniform composition throughout, or are there gradients?

4. How good are our models of stars? Are there important parameters that we have failed to take into account?

5. What counts when we see differences between stars? For example, minor differences in activity levels may just reflect different present states within near-identical long-term behavior. Current differences in lithium abundances may be a manifestation of past differences in angular momentum, but they may not imply any real difference in the present structure or evolutionary state.

6. Can rotation alter the macro-state of an STS, or is its influence confined to micro-effects?

\section{Stay Tuned}

Among works in progress, we note the following as pertinent to the subject matter of this paper. First, the present authors have started a survey of activity in 5000 to 8000 STSs within 60 pc. The sample is defined from Hipparcos 
results, and the observations consist of $R=2000$ spectra that include the $\mathrm{Ca}$ II $\mathrm{H}$ and $\mathrm{K}$ lines. We expect to determine metallicities and gravities as well from these spectra.

Second, these data and more will form the basis for a database on nearby stars. This database is being supported by NASA and NSF, in part from recognition that the nearest stars need full characterization in order to work as targets for missions such as SIM (the Space Interferometry Mission) and TPF (the Terrestrial Planet Finder).

Third, although somewhat removed in subject, and greatly removed in distance, an intriguing Cycle 8 HST program will obtain photometry of extremely high precision for stars in the core of 47 Tucanae, with the goal of detecting planetary transits. The observations should also say a lot about intrinsic variability in very old stars of about one solar mass, albeit of low metallicity.

Acknowledgments. We wish to acknowledge ongoing collaborations with many individuals who have played a part in what is described here. They include Burt Jones, John Stauffer, Dave Latham, Todd Henry, Lionel Siess, Sallie Baliunas, Bob Donahue, Fritz Benedict, Otto Franz, Marc Pinsonneault and Don Terndrup.

A fuller version of this presentation may be found at: http://www.lowell.edu/users/jch/workshop/drs/drs-p1.html

\section{References}

Gray, D. F. 1994a, ApJ, 428, 765

Gray, D. F. 1994b, PASP, 106, 1248

Cayrel, R., Perrin, M.-N., Barbuy, B. \& Buser, R. 1991a, A\&A, 247, 108

Cayrel, R., et al. 1991b, A\&A, 247, 122

Jones, B. F., Fischer, D. \& Soderblom, D. R. 1999, AJ, 117, 330

Olsen, E. H. 1988, A\&A, 189, 173

Olsen, E. H. 1993, A\&AS, 102, 89

Pinsonneault, M., et al. 1998, ApJ, 504, 170

Soderblom, D. R., et al. 1998, ApJ, 504, 192

Vaughan, A. \& Preston, G. W. 1980, PASP, 92, 385 